\title{
Techniques for Image Segmentation: A Critical Review
}

\author{
Harshita Mishra, Anuradha Misra
}

\begin{abstract}
In today's world there is requirement of some techniques or methods that will be helpful for retrieval of the information from the images. Information those are important for finding solution to the problems in the present time are needed. In this review we will study the processing involved in the digitalization of the image. The set or proper array of the pixels that is also called as picture element is known as image. The positioning of these pixels is in matrix which is formed in columns and rows. The image undergoes the process of digitalization by which a digital image is formed. This process of digitalization is called digital image processing of the image (D.I.P). Electronic devices as such computers are used for the processing of the image into digital image. There are various techniques that are used for image segmentation process. In this review we will also try to understand the involvement of data mining for the extraction of the information from the image. The process of the identifying patterns in the large stored data with the help of statistic and mathematical algorithms is data mining. The pixel wise classification of the image segmentation uses data mining technique.
\end{abstract}

Index Terms - computer vision, digital image processing, data mining, mathematical algorithm

\section{INTRODUCTION}

In the field of computer vision, the division of the image into small segments is called image segmentation. The image for the segmentation process is digital in nature. The importance of the image segmentation is to obtain more specific, accurate, easy to understand and easy to analyze image.

The collection of the segment of the image makes complete image. The pixels of a segment of the image are similar with respect to the color of the pixels, texture of the pixels and intensity of the pixels.

Various techniques of the image segmentation are mentioned below-

a) Edge based segmentation- the process of edge detection is well developed technique in the field of the image processing. The edges that are identified by the edge detection technique are disconnected. For segmentation

Manuscript revised on April 1, 2021 and published on April 10, 2021 Harshita Mishra, Department of Computer Science and Engineering, ASET Amity University, Lucknow, Uttar Pradesh, India Email id: mharshu1995@gmail.com

Anuradha Misra, Department of Computer Science and Engineering, ASET Amity University, Lucknow, Uttar Pradesh, India Email id: amisra@lko.amity.edu of the object from the image a closed region boundary is required. The edge based segmentation can be applied on the information granules that are crisp pixel region.

b) Fuzzy theory based segmentation- In the fuzzy based segmentation of the image there is use of the morphological opening and closing of the operation that smoothen the image for performing gradient operations on the final image.

c) PDE based segmentation- A non-linear discontinue method known as partial differential equation. This is the method in which level is set for the gray image. This method is fast and effective.

d) ANN based segmentation- The use of neural network for separating the targeted image. For performing this segmentation dataset for the image is used. The noise is removed from the image this step is used as pre-processing step for performing segmentation of the image.

e) Threshold based segmentation- The simplest method for the segmentation of the image is threshold based segmentation. This method is related to the threshold value known as clip-level. This changes grey-scale image to binary image. By this segmentation method we get more optimized outcomes.

f) Region based segmentation- In this segmentation process the join of the edges and region used for obtaining information. It uses method morphological watershed algorithm. The extractions of the features are more accurate by this segmentation method in comparison to the other segmentation method.

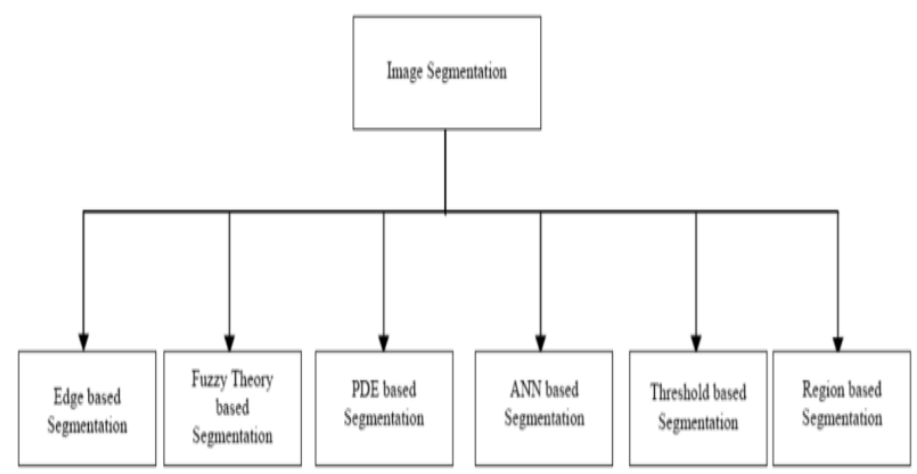

Fig 1 Type of Image Segmentation

\section{A. Application-}

Various application of the image segmentation process is image retrieval based on the content, medical study, object detection, face detection, pattern recognition, fingerprint recognition, segmentation of the video object and video 
surveillance.

\section{RELATED WORK}

In [1] the author has discussed about different techniques and methods of image segmentation those are useful in the different fields of the science such as in the medical science, for the recognition process of the object and pattern in the image. The techniques described by the author are-

1) Image segmentation based on edge detection

* Roberts edge detection

* Sobel edge detection

* Prewitt edge detection

2) Image segmentation based on thresholding method

- Global thresholding method

- Variable thresholding method

- Multiple thresholding method

3) Image segmentation based on region

- Region growing method

- Region splitting and merging

4) Image segmentation based on clustering

- K-mean

- Fuzzy K-mean

In [2] the author of the paper has described about approaches for segmentation of the image that are classified as-

a) Local segmentation of the image and

b) Global segmentation of the image.

There are two approaches of the image segmentation on the basics of the properties of the image. Those approaches are

i. Discontinuity detection approach for image segmentation

ii. Similarity detection approach for image segmentation

In [3] the author has presented the work in which the author has explained the use of image segmentation in the field of the ultrasonic for the accuracy in the detection of the damage in the c-scan of the complex image. The techniques of the image segmentation that are used for the C-Scan in ultrasonic testing are-
a) Threshold technique
b) Edge detection technique
c) Region based technique
d) Clustering technique

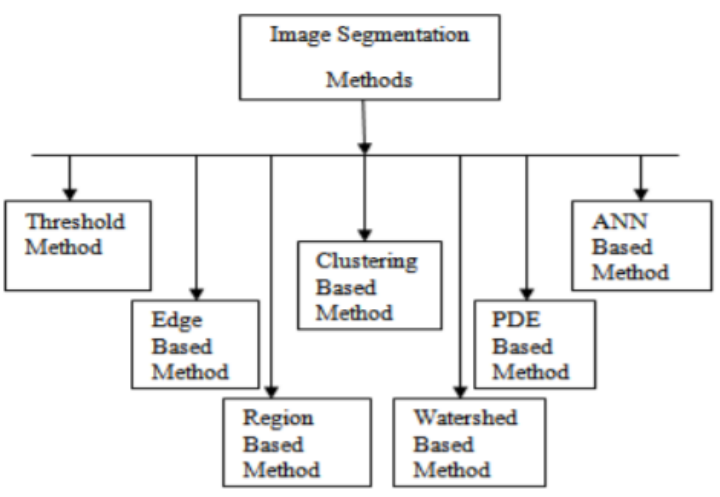

Fig 2- Image Segmentation Technique
In [4] the author has discussed about the use of image segmentation for the deep learning in the field of the M.R.I. The ventricle segmentation is used in the field of MR and ultrasound. The important role of the ventricle segmentation is to describe the innermost tissue or layer and membrane that are attached to the wall of the heart of LV/RV. This segmentation is also important for obtaining clinical details such as LVEDV that is left ventricular end-diastolic volume. In [5] the author has specially focused on the techniques of clustering for the segmentation of the image. The process of clustering has made segmentation of the image more effective. Different clustering techniques are-
a) Relevance feedback clustering
b) Log based clustering
c) Hierarchical clustering
d) Retrieval dictionary based clustering
e) K-mean algorithm clustering
f) $\mathrm{N}$-cut algorithm clustering

In [6] the author explained different techniques for the segmentation of the image. In the review work the author has come to outcome that there is no prefect technique for the segmentation of the image. The method for the segmentation of the image is based on-
a) Pixel of the image
b) Color of the image
c) Texture of the image
d) Intensity of the image
e) Similarity of the image
f) Content of the image
g) Problem domain of the image

In [7] the author has explained the use of image segmentation algorithms for detection of the disease in the plants leaf. The techniques of the image segmentation are tested on the plants such as banana, beans, jackfruit, lemon, mango, potato, tomato and sapota.

In [8] the author has explained the use of the segmentation of the image in the field of the medical science. The segmentation process is used for medical therapy device. The segmentation of the image saves lots of time and is a good replacement for the manual drawing by the surgeon. In medical science the segmentation process is categorized as automatic and semi-automatic techniques of image segmentation or they are known as bottom-up segmentation and top-down segmentation of the image. In automatic segmentation of the image there is no human involvement for the segmentation process of the image. In automatic segmentation slit and merge is involved. In semi-automatic segmentation there is human interact for the segmentation process of the image.

In [9] the author has explained that there is no global technique for the segmentation of the image. For every image different technique is required for the segmentation process of the image. The author divides segmentation process as block image segmentation method. The segmentation of the image undergoes several decisions making. In the field of the image processing the image segmentation process is highly challenging process.

In [10] the author has presented a technique of image segmentation that is known as shape-based segmentation of 
the image. This segmentation is also known as fuzzy clustering for image segmentation using generic shape information. By fuzzy clustering method of segmentation high-rated quality of the segmentation of the image is performed.

In [11] the author has focused on two methods pre-processing of the image and segmentation of the image. The use of filters such as histogram, gabour helps in improving the different pre-processing methods of the image. The segmentation methods are known to be simplest method for segmentation of the image such as thresholding, region based method but there application is very less. For the improvement of the technique other techniques are merged such as fuzzy C-mean. In [12] the author has explained that for analysis of the image, segmentation is the basis step that is low level processing to digital image processing. The basis of the image segmentation is related to the grey level image. The extension for the grey level segmentation is color segmentation of the image. There are few grey level segmentation method that can't be directly transformed to the color segmentation of the image.

In [13] the author tells that for the study of the signal processing technique and applications image segmentation is used. The use of the segmentation is to find shape and position of the image according to appearance of the information. The use of the segmentation of the image is to find out objects for object-based measurement that is shape and size and in object-based video compression for identifying object in moving scene.

In [14] the author has described the system for adaptive color image segmentation. The use of adaptive multiple levels if thresholding method for segmentation of the image is explained by the author. Several tests were performed on different images using ACIS.

A

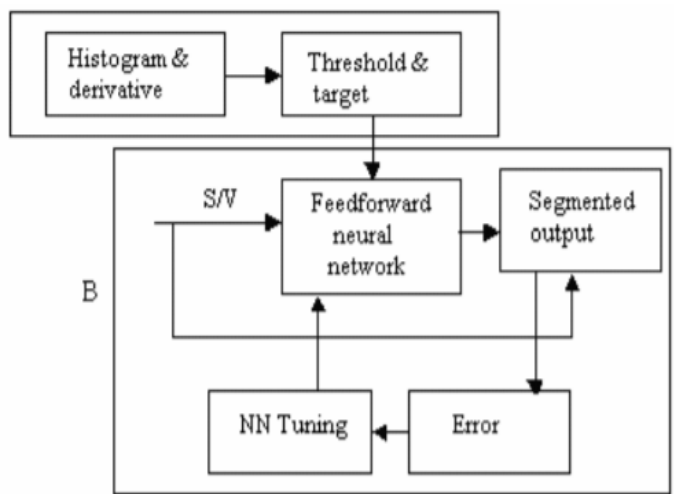

Fig 3 (A) Adaptive threshold bloc (B) Neutral network segmentation block

In [15] the author has referred unsupervised method such as cluster based algorithm for the segmentation of the image. Different cluster based algorithm were examined on different images. These algorithms are k-mean, improved k-mean, fuzzy c mean, improved fuzzy c mean. The k-mean algorithm takes less time for the result of the segmentation for the image but gives poor results.

\section{CONCLUSION}

In this review paper we have referred through different papers published by different authors. In these papers different authors have explained various methods, algorithms and techniques for the image segmentation. The use of the image segmentation process in the different field of the present such as in the medical science, in identifying different diseases in the plants leaf, finding specific shapes and figures from the image etc. are also explained.

In the coming time the use of the image segmentation is going to be very important for any kind of research work and in finding results to the problems.

For the better image segmentation results, the use of clustering technique is very helpful. It increases the efficiency of the information retrieval process.

We came to conclusion that there is no specific method for image segmentation but for the proper segmentation process we need to use hybrid of two or more techniques of image segmentation for better results.

The authors have given us the extension for the grey level image segmentation that is use of color image segmentation that provides us rich information related to the image.

\section{FUTURE WORK}

In future work of our study we will explore on new techniques of the image segmentation. The techniques that are described by the other authors have some disadvantage. They are not very specific in extracting information from the image. So we will focus on the techniques of the image segmentation that can give best results. The results will be useful for the different fields such as in the area computer vision, medical science and knowing color, shape, texture of the image. In the upcoming work we will study how hybrid of image segmentation technique can be useful for receiving best version of the outcome that can be more useful for solving problems.

\section{CONFLICT OF INTEREST}

The authors declare no conflict of interest.

\section{REFERENCES}

[1] Kaur, A. (2012). A review paper on image segmentation and its various techniques in image processing. International Journal of Science and Research.

[2] Kaur, D., \& Kaur, Y. (2014). Various image segmentation techniques: a review. International Journal of Computer Science and Mobile Computing, 3(5), 809-814.

[3] Wronkowicz-Katunin, A., Mihaylov, G., Dragan, K., \& Timofiejczuk, A. (2019). Uncertainty estimation for ultrasonic inspection of composite aerial structures. Journal of Nondestructive Evaluation, 38(3), 1-20.

[4] Chen, C., Qin, C., Qiu, H., Tarroni, G., Duan, J., Bai, W., \& Rueckert, D. (2020). Deep learning for cardiac image segmentation: A review. Frontiers in Cardiovascular Medicine, 7, 25.

[5] Thilagamani, S., \& Shanthi, N. (2011). A survey on image segmentation through clustering. International Journal of Research and Reviews in Information Sciences, 1(1), 14-17.

[6] Khan, M. W. (2014). A survey: Image segmentation techniques. International Journal of Future Computer and Communication, 3(2), 89.

[7] Singh, V., \& Misra, A. K. (2017). Detection of plant leaf diseases using image segmentation and soft computing techniques. Information processing in Agriculture, 4(1), 41-49. 


\section{Available online at www.ijrat.org}

[8] Zanaty, E. A., \& Ghoniemy, S. (2016). Medical image segmentation techniques: an overview. International Journal of informatics and medical data processing, 1(1), 16-37.

[9] Zaitoun, N. M., \& Aqel, M. J. (2015). Survey on image segmentation techniques. Procedia Computer Science, 65, 797-806.

[10] Ali, M. A., Karmakar, G. C., \& Dooley, L. S. (2008). Fuzzy Clustering for Image Segmentation Using Generic Shape Information. Malaysian journal of computer science, 21(2), 122-138.

[11] Jeyavathana, R. B., Balasubramanian, R., \& Pandian, A. A. (2016). A survey: analysis on pre-processing and segmentation techniques for medical images. International Journal of Research and Scientific Innovation (IJRSI).

[12] Kumar, P. P., Pabboju, D. S., \& Sekharaiah, D. K. C. (2018). An Effective Image Segmentation Method For Region Based Image Retrieval System. International Journal of Pure and Applied Mathematics, 118(24)

[13] Aly, A. A., Deris, S. B., \& Zaki, N. (2011). Research review for digital image segmentation techniques. International Journal of Computer Science \& Information Technology, 3(5), 99.

\section{AUTHORS PROFILE}

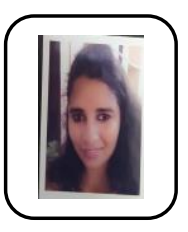

Harshita Mishra is M.tech student in Computer Science and Engineering in Amity University, Lucknow, Uttar Pradesh. The area of interest digital image processing, image segmentation, artificial intelligence

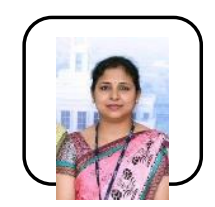

Dr. Anuradha Misra is Assistant Professor of Amity University in the department Amity School of Engineering and technology in Lucknow, Uttar Pradesh. The area of interest is data mining, database management system, operating system.
[14] Deshmukh, K. S., \& Shinde, G. N. (2006). An adaptive color image segmentation. ELCVIA Electronic Letters on Computer Vision and Image Analysis, 5(4), 12-23.

[15] Sathya, B., \& Manavalan, R. (2011). Image segmentation by clustering methods: performance analysis. International Journal of Computer Applications, 29(11), 27-32. 